\title{
Construction of Stochastic Model for Time to Dengue Transmission with Normal Distribution
}

\author{
K.L. Muruganantha Prasad, P.S. Stem Edilber, \\ R. Subramoniam
}

\begin{abstract}
In this paper deals with the study of stochastic model for predicting the time to dengue transmission. As the immune capacities of an individual differ and also have its personal resistance, the antigenic diversity threshold is dissimilar for different person. We construct a model to find the number of people affected bydengue (2016-2017) using normal distribution.
\end{abstract}

\section{INTRODUCTION}

Dengue fever (DF) and Dengue Hemorrhagic fever(DHF) collectively known as dengue are mosquito borne, potentially mortal, flu-like viral disease that affect humans worldwide. Transmitted to humans by the bite of an infected mosquito Aedesageypti, dengue is caused by one of four serotypes termed as DENV-1,DENV-2,DENV-3 and DENV-4 or antigen-specific viruses dengue virus is a part of flaviviridae family. Dengue is one of the most rapidlyspreading mosquito-borne viral disease in the world and inflicts significant health, economic and social burdens on population.

Mathematical and statistical models describing the transmission of dengue viruses appeared in the study of observations related to pathogenesis of dengue hemorrhagic fever and providing a better understanding of the nature and dynamics of the transmission of dengue infection as well as outbreaks and stimulate the impact of control strategies in disease transmission. The time to dengue viruses transmission by biting mosquito alone is the only mode of dengue transmission. The bites of mosquitos are assumed between seropositive person who is labeled as index case and seropositive state takes place over an incubation period due to the contraction of dengue to the partner from the index case by the bites of mosquitoes.

In this paper we find the number of people affectedby dengue(2016-2017) by using normal distribution.

\section{NORMAL DISTRIBUTION}

The normal distribution describes a family of continuous probability distributions, having the same general shape, and differing in their location (that is, the mean or average) and scale parameters (that is, the standard deviation). The graph of its probability density function is a symmetric and bellshaped curve. The development of the general theories of the normal distributions began with the work of de Moivre $(1733,1738)$ in his studies of approximations to certain

Revised Manuscript Received on July 10, 2019.

K.L. Muruganantha Prasad, Department of Mathematics, H.H. The Rajahs College, Pudhukottai, Tamilnadu, India.

P.S. Stem Edilber, Research Scholar, H.H. The Rajahs College, Pudhukottai, Tamilnadu, India.

R. Subramoniam, Department of Mathematics, Lekshmipuram College of Arts and Science, Neyyoor, Tamilnadu, India. binomial distributions for large positive integer $n>0$. Further developments continued with the contributions ofLegendre(1805),Gauss(1809),Laplace(1812),Bessel(1818, 1838),Bravais(1846), Airy (1861), Galton (1875, 1889), Helmert (1876), Tchebyshev (1890), Edgeworth (1883, 1892, 1905), Pearson (1896), Markov (1899, 1900), Lyapunov (1901), Charlier (1905), and Fisher (1930, 1931), among others. For further discussions on the history of the normal distribution and its development, readers are referred to Pearson (1967), Patel and Read (1982), Johnson et al. (1994), and Stigler (1999), and references therein. Also, see Wiper et al. (2005), for recent developments. The normal distribution plays a vital role in many applied problems of biology, economics, engineering, financial risk management, genetics, hydrology, mechanics, medicine, number theory, statistics, physics, psychology, reliability, etc., and has been has been extensively studied, both from theoretical and applications point of view, by many researchers, since its inception.

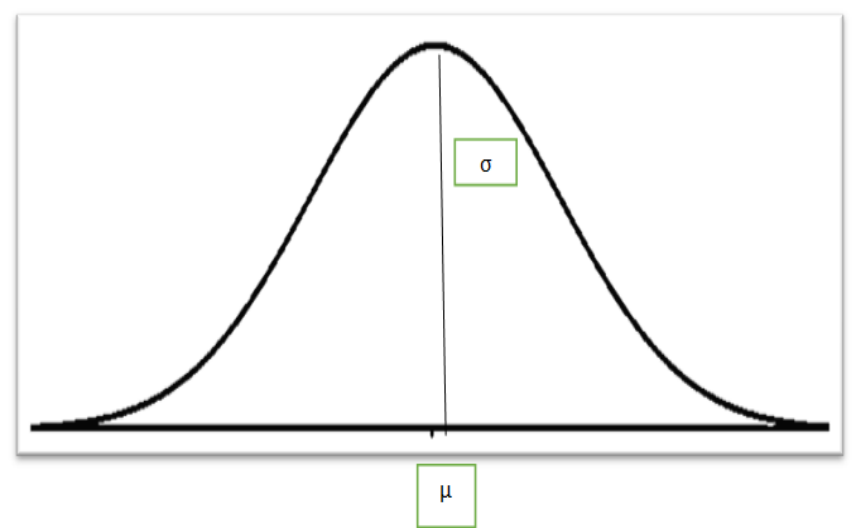

Fig. 1: Normal Distribution Curve

\section{Definition}

A continuous random variable $\mathrm{X}$ is said to have a normal distribution with mean $\mu$ and variance $\sigma^{2}$ that is $\mathrm{X} \sim \mathrm{N}(\mu$, $\left.\sigma^{2}\right)$ if its pdf $f_{x}(x)$ and $\operatorname{cdf}_{X}(x)=P(X \leq x)$ are respectively given by

$$
f_{x}(x)=\frac{1}{\sigma \sqrt{2 \pi}} e^{-(x-\mu)^{2} / 2 \sigma^{2}}-<x<\infty
$$

and

$$
F_{X}(x)=\frac{1}{\sigma \sqrt{2 \pi}} \int_{-\infty}^{x} e^{-(y-\mu)^{2} / 2 \sigma^{2}} d y
$$

$=1 / 2[1+\operatorname{erf}(\mathrm{x}-\mu / \sigma \sqrt{2})]-\infty<x<\infty-\infty<\mu<\infty$, $\sigma>0 \ldots(2)$

\section{Published By:}

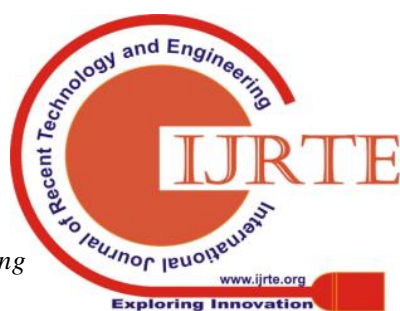


Where erf(.) denotes error function and $\mu$ and $\sigma$ are location and scale parameters respectively

In the above definition if $\mathrm{X} \sim \mathrm{N}(0,1)$ then it is called as standard normal distribution.

\section{ASSUME \& RESULTS}

$\boldsymbol{\mu}$ is the number of people having dengue positive $\boldsymbol{\sigma}$ is number of people having dengue negative.

Note

$\mathrm{X}$ has a normal distribution if and only if $z=\frac{x-\mu}{\sigma}$ has a $\mathrm{N}(0,1)$ distribution.

We know that many practical applications are involve normal distributions. Normal pdf's however contain some factor such as $\left\{s^{2}\right\}$. Hence their antiderivatives cannot be obtained in closed form and numerical intergration techniques must be used. Because of relationship between normal and standard normal variables we need only compute probabilities for standard normal random variables. To denote the cdf of a standard normal variable $\mathrm{Z}$ by

$\varphi(z)=\int_{-\infty}^{z} 1 / \sqrt{2 \pi} \exp \left\{\frac{-w^{2}}{2}\right\} d w$

Let $X$ have a $\mathrm{N}\left(\mu, \sigma^{2}\right)$ distribution . suppose we want to compute $F_{X}(x)=P(X \leq x)$ for a specified $\mathrm{x}$. for $\mathrm{Z}=(\mathrm{x}-\mu) / \sigma$ expression (3) implies

$$
F_{x}(x)=P(X \leq x)=P\left(X \leq x-\frac{\mu}{\sigma}\right)=\varphi\left(x-\frac{\mu}{\sigma}\right)
$$

Thus we only need numerical integration computations for $\varphi(x)$. Normal quantiles can also be computed using quantiles based on $\mathrm{z}$.

\section{To find mean and variance}

Consider the pdf

$$
f(x)=\frac{1}{\sigma \sqrt{2 \pi}} e^{-(x-\mu)^{2} / 2 \sigma^{2}}
$$

Where mean $=\mu$

Variance $=\sigma^{2}>0$

Standard deviation $=\sigma>0$

1. To prove $\int_{-\infty}^{\infty} f(x) d x=1$

Assume that $\int_{-\infty}^{\infty} e^{-x^{2}} d x=\sqrt{\pi}$

Now $\int_{-\infty}^{\infty} f(x) d x=\frac{1}{\sigma \sqrt{2 \pi}} e^{-(x-\mu)^{2} / 2 \sigma^{2}} d x$

$\mathrm{y}=\mathrm{x}-\mu / \sqrt{2} \sigma$

$\mathrm{dy}=\mathrm{dx} / \sqrt{2} \sigma$

$\mathrm{dx}=\sqrt{2} \sigma \mathrm{dy}$

$=\frac{1}{\sigma \sqrt{2 \pi}} \int_{-\infty}^{\infty} e^{-y^{2}} \sqrt{2} \sigma d y \quad=1$

2. To prove $\mathbf{E}(\mathrm{X})=\boldsymbol{\mu}$

$\mathrm{E}(\mathrm{X})=\int_{-\infty}^{\infty} x f(x) d x$

$=\int_{-\infty}^{\infty} \frac{1}{\sigma \sqrt{2 \pi}} \mathrm{x} e^{-(x-\mu)^{2} / 2 \sigma^{2}} d x$

$\mathrm{y}=\mathrm{X}-\mu$

$\mathrm{dy}=\mathrm{dx}$

$\mathrm{x}=\mathrm{y}+\mu$

$=\mu+\frac{1}{\sigma \sqrt{2 \pi}} \mathrm{y} e^{-y^{2} / 2 \sigma^{2}} d y$

$=\mu$ where $\frac{1}{\sigma \sqrt{2 \pi}} \mathrm{y} e^{-y^{2} / 2 \sigma^{2}} d y 0$

3. To prove $\operatorname{Var}(X)=\sigma^{2}$

$$
\begin{aligned}
& \operatorname{Var}(\mathrm{X})=\mathrm{E}\left((x-\mu)^{2}\right) \\
& \mathrm{E}\left((x-\mu)^{2}\right)=\int_{-\infty}^{\infty}(x-\mu)^{2} f(x) d x \\
& =\frac{1}{\sigma \sqrt{2 \pi}} \int_{-\infty}^{\infty}(x-\mu)^{2} e^{-(x-\mu)^{2} / 2} \sigma^{2} d x
\end{aligned}
$$

$$
\begin{aligned}
& \begin{array}{l}
\mathrm{y}=\mathrm{x}-\mu \\
\mathrm{dy}=\mathrm{dx}
\end{array} \\
& \mathrm{x}=\mathrm{y}+\mu \\
& \\
& \qquad \frac{1}{\sigma \sqrt{2 \pi}} \int_{-\infty}^{\infty}(y)^{2} e^{-(y)^{2} / 2} \sigma^{2} d y \\
& =\sigma^{2}
\end{aligned}
$$

\section{Example}

1. During the year (2016-2017) the total number of patients admitted as dengue 430, in Asaripallamgovt hospital in that 120 has dengue positive and 310 has dengue negative.

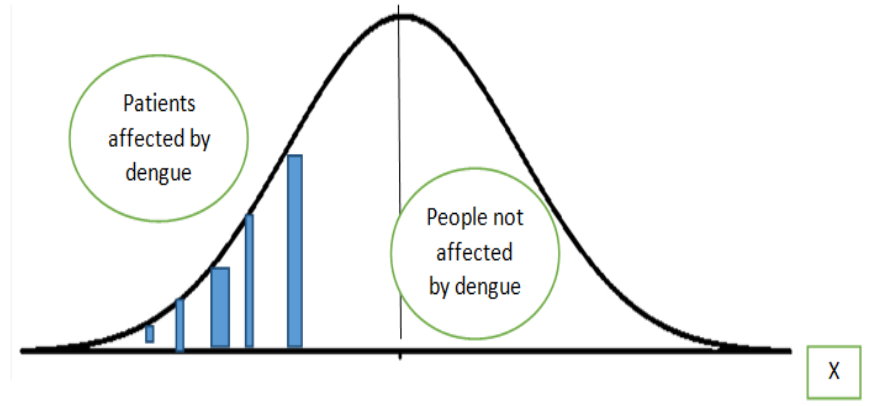

Fig. 2: Total dengue affected and unaffected peoples in As aripallamgovt hospital(2016-2017)

Table 1: Shows the number of people affected by dengue in government Hospital (2016-2017)

\begin{tabular}{|l|l|l|l|l|}
\hline S.No & Hospital & $\begin{array}{l}\text { No of } \\
\text { patients }\end{array}$ & $\begin{array}{l}\text { Dengue } \\
\text { Positive }\end{array}$ & $\begin{array}{l}\text { Dengue } \\
\text { Negative }\end{array}$ \\
\hline 1 & $\begin{array}{l}\text { Govt hospital } \\
\text { Asaripallam }\end{array}$ & 430 & 120 & 310 \\
\hline 2 & $\begin{array}{l}\text { Govt Hospital } \\
\text { Thuckalay }\end{array}$ & 327 & 92 & 235 \\
\hline 3 & $\begin{array}{l}\text { Govt Hospital } \\
\text { Colachel }\end{array}$ & 230 & 42 & 188 \\
\hline 4 & $\begin{array}{l}\text { Govt Hospital } \\
\text { Marthandam }\end{array}$ & 143 & 23 & 120 \\
\hline 5 & $\begin{array}{l}\text { Govt Hospital } \\
\text { sundavillai }\end{array}$ & 67 & 33 & 34 \\
\hline
\end{tabular}

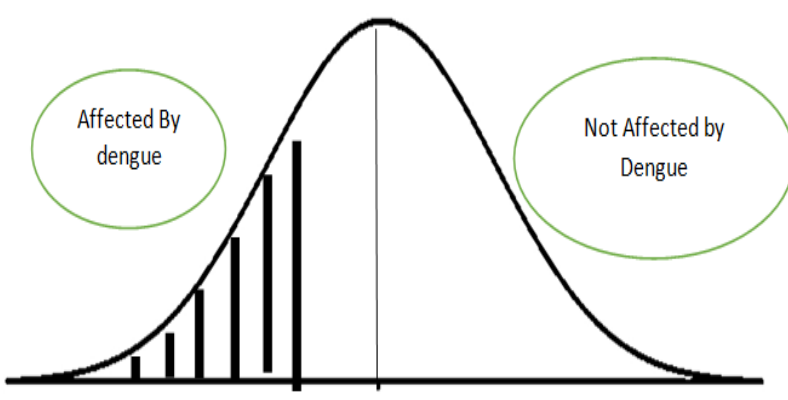

Fig. 3: Number of people affected by dengue and unaffected by dengue (2016-2017) 


\section{REFERENCES}

1. Ahsanullah et al normal and Students t distributions and their applications, Atlantis Studies in probability and statistics 4 DOI 10.2991/978-94-6239-4-2, Atlantis Press and the authors 2014.

2. Applied statistics and probability for engineers by Montgomery and rungers.

3. Probability and Statistics for Engineering and the sciences by jeydevore.

4. Introduction to probability models by Sheldon. M. ross.

5. Medical reports from govt hospital 2016-2017.

6. Medical News Today Nov 2018.

7. Operational Research theory and applications By Taha. 